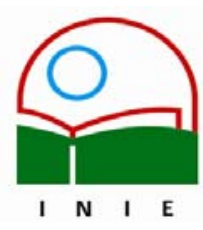

Universidad de Costa Rica

Facultad de Educación

Instituto de Investigación en Educación

ACTUALIDADES INVESTIGATIVAS EN EDUCACION

\title{
LA REVOLUCIÓN CIENTÍFICO-TÉCNICA Y LA EDUCACIÓN ${ }^{1}$
}

\section{Dr. Arnoldo Mora²}

Al igual que la Naturaleza, según la física cuántica, la historia de la civilización procede y evoluciona mediante saltos cualitativos que solemos llamar "revoluciones". Una revolución consiste en un cambio muy rápido en sus manifestaciones visibles, si bien posee raíces de largo alcance que actúan de manera casi imperceptible a través de largos periodos de tiempo. Pero lo propio y más llamativo de una revolución es que acelera los cambios en lapsos de tiempo muy cortos y estos cambios se operan en todos los ámbitos del actuar humano.

Sin embargo, si bien estos cambios son radicales y vertiginosos, no actúan de manera desordenada. Dentro de este aparente caos, se da un orden, no solo cronológico sino, sobre todo, causal en sus manifestaciones, cuya consecuencias concretas configuran las diversas culturas a través de la historia de la humanidad. Estos cambios cualitativos o "saltos dialécticos", definen el ser y quehacer del ser humano en el transcurso del tiempo. Desde el punto de vista biológico, es decir, anatómico y fisiológico, lo que distingue a la especie sapiens de las otras especies vivientes es el tamaño y complejidad de su cerebro. Ninguna otra especie conocida posee 14 centímetros cúbicos de masa encefálica. Por eso podemos decir que la obra maestra de la evolución, la obra maestra del Cosmos, es nuestro cerebro, culminación de la evolución de la vida sobre el universo conocido hasta el presente. Cuidar y cultivar nuestro cerebro, hacer un uso adecuado del mismo, descubrir sus potencialidades y ponerlas al servicio de las mejores causas, del lado de los mas altos valores humanos, constituye la razón de ser, la justificación de nuestra existencia, del corto paso de los individuos por la vida y de los pueblos y de las civilizaciones a través de la historia. La manera cómo los diversos pueblos en sus diversas culturas han logrado cultivar ese acervo de

\footnotetext{
1 Conferencia Congreso Internacional de Investigación Educativa: "Investigar para transformar" Instituto de Investigación en Educación-Universidad de Costa Rica, COSTA RICA. 3 de febrero de 2005.

2 Doctor en Filosofía de la Universidad de Lovaina, Bélgica. Se ha desempeñado en importantes cargos como: Ministro de Cultura, Juventud y Deportes; Decano de la Facultad de Letras, Director y Subdirector de la Escuela de Filosofía de la Universidad de Costa Rica; Catedrático de la Universidad de Costa Rica y de la Universidad Nacional, ambas universidades estatales.
} 
valores, conservándolos y transmitiéndolos de generación en generación mediante tradiciones e instituciones sociales, es la tarea que han cumplido los sistemas educativos en las diversas sociedades humanas que han existido a través de la historia. Pero el ser humano al nacer, precisamente porque su cráneo es de un tamaño superior relativamente al de todas las otras especies, no puede permanecer, como el resto de los mamíferos, mucho tiempo en el seno materno. Por eso nace demasiado pronto. Todo ser humano es un prematuro, por lo que el cerebro debe crecer en los primeros años de vida rápidamente. Es en este tiempo cuando aprende a hablar, actividad que le ha permitido obtener la superioridad sobre el resto de las otras especies. El habla es la expresión material del pensamiento. La actividad pensante consiste en convertir los objetos de la percepción sensorial en símbolos sonoros. Eso es lo propio del pensar humano.

Es gracias a la construcción de un universo simbólico que el ser humano interpreta y modifica su entorno, le da un sentido a su existencia sobre la tierra y construye una realidad objetiva pero no natural puesto que es producto de su acción. Esa realidad objetiva es tanto material como social, es decir, consiste tanto en construir objetos materiales para responder a sus necesidades vitales, como hacer una guarida para protegerse de las inclemencias del clima o fabricar herramientas para obtener alimentos o defenderse, o establecer relaciones sociales para hacer funcional el centro de esas relaciones sociales que conformen el grupo en cuyo seno nace y se desenvuelve.

Para ambas funciones responde el sistema educativo. Si bien el ser humano trae consigo al nacer una herencia genética, sin embargo esta no es suficiente para sobrevivir. Debe aprender a hacerlo dentro de las circunstancias en que se desenvuelve la vida del grupo. Debe aprender, ante todo, una lengua, la que por eso se llama "lengua materna", y con ello, comunicarse con su entorno humano y aprender a interpretar y actuar en su torno material y cultural. En consecuencia, el ser humano es tanto un ser biológico, como un ser social y cultural. De su medio recibe un conjunto de significaciones para la vida.

Esas significaciones las llamamos "valores". Transmitir valores, como base del fundamento de toda la conducta, constituye la esencia misma de la acción educativa. Educar es inculcar valores, es establecerlos como normas de comportamiento o principios por los cuales regir la vida y juzgar la de los demás. La herencia genética provee potencialidades, pero es la educación, es decir, lo que solemos llamar "cultura", lo que convierte esas potencialidades en acciones y actitudes concretas. 
El valor fundamental sobre el que se basa todo otro valor, lo que podríamos llamar el valor matricial del que proceden todos los demás y al que se ordenan, es la vida. La vida posee dos facetas: preservarla y trasmitirla, tanto física como culturalmente. Por eso decimos que vivir es ante todo, sobrevivir y convivir. El sobrevivir responde a la necesidad de preservar la vida, tanto propia como de los seres que nos son queridos. Es, por ende, exigir el reconocimiento de su propio valor ante los otros, sean estos fuerzas externas de la Naturaleza, sean fuerzas sociales o humanas. Convivir por el contrario, es reconocer el derecho de los otros a vivir. El primer caso implica cierto nivel de violencia pues no se es viviente sin más, hay que ganarse el derecho a la vida como valor supremo. El segundo, por el contrario, exige ponerle límites a esa violencia.

A esto lo solemos llamar "ética y moral", ética como conjunto de normas que deben regir nuestra conducta, y moral como su aplicación dentro de un contexto social dado. La ética es la actividad humana mediante la cual humanizamos la violencia poniéndole límites, justificándola dentro de esos límites y condenándola cuando de excede esos límites. Esto lo establece la ética en virtud de un principio fundamental y fundante de toda ética: el principio de legítima violencia. La violencia no es ni buena ni mala en sí misma; lo que la hace buena o mala es el uso que de la misma hacemos dentro de determinadas circunstancias. Es buena la violencia defensiva, es decir, que se usa como medio de preservar un derecho legítimo que se ve injustamente amenazado; es mala toda violencia agresiva, es decir, que conculca el derecho legítimo de otros.

Por eso toda acción humana tiene como objetivo preservar y desarrollar la vida en todas sus auténticas manifestaciones. Esto lo hace el ser humano mediante la acción práctica. La actividad básica del ser humano es el trabajo mediante el cual transforma el mundo material que lo rodea, con lo que logra crear condiciones objetivas que le permitan vivir.

Toda la actividad práctica implica un proceso que consta de tres momentos. Ante todo, la actividad que responde a la satisfacción de necesidades vitales. Cuando esta actividad cumple con sus finalidades el ser humano se vuelve conservador en la medida que no hace sino repetir esa actividad en vista de que lo que busca son resultados satisfactorios. Pero cuando esta actividad no produce los resultados apetecidos, cuando se descubre un error o se sufre un fracaso, entonces viene el segundo momento o etapa crítica.

Es allí donde el ser humano alcanza su madurez como ser humano, porque la experiencia del error o la conciencia del fracaso son el suelo 
nutricio donde brota fecundo el pensamiento y la conciencia reflexiva. Es entonces cuando emerge la razón como capacidad del ser humano de formular preguntas radicales, es decir, que van al fondo de los problemas. Ansiosos nos interrogamos por qué hemos fracasado si hasta ahora todo había caminado bien.

Es allí cuando se constata que la sola experiencia no basta, que el criterio de empiricidad pura no es suficiente y que la verdad es algo mas que la acumulación y clasificación, por mas lógicamente ordenada según criterios de causalidad que sea, no basta. La razón busca explicaciones, formula teorías, va más allá de lo real inmediato para verlo a la luz de lo posible. Descubre el carácter imprescindible de la dimensión especulativa del saber humano, como diría Karl Popper. Toda la ciencia no es mas que el intento por responder a la pregunta radical por excelencia y que podemos formular con este interrogante: ¿Cómo es posible que se haya dado esto o lo otro y, en última instancia, cómo es posible que lo real exista? O, para decirlo en el lenguaje de La Metafísica de Aristóteles, es preguntarse por "el ser del ente".

La inteligencia humana es producto de la conciencia de nuestros errores y fracasos o, para decirlo en palabras de Sócrates, solo la conciencia existencial de nuestra ignorancia es el punto de partida del saber. Pero la reflexión especulativa no es primera. La ciencia nace de la exigencia práctica de superar la ignorancia, de vencer el error y de superar el fracaso. Las grandes revoluciones en la historia de la civilización humana han sido logradas gracias a los saltos que el ser humano ha dado en el tiempo para vencer los obstáculos que le impiden disfrutar de una sobrevivencia y una convivencia mejores.

La interpretación teórica, es decir, simbólica y, por ende, potencial de la realidad circundante, la llamamos "ciencia" como saber el porqué como decía Aristóteles. Pero todo saber o conocimiento no es más que poder potencial. Todo conocimiento científico trae aparejado un acrecentamiento $\mathrm{d}$ el poder de quien la posee. Este poder se manifiesta mediante la construcción de objetos que nos permiten dominar nuestro entorno tanto material como social. Esto quiere decir que todo saber engendra poder económico y poder político, esto es, autoridad sobre los otros.

El conjunto de medios o instrumentos materiales que prolongan y acrecientan el poder lo llamamos técnicas. Cuando la ciencia o saber científico produce medios materiales y, a su vez, estos medios materiales o instrumentos son parte indisociable del conocimiento científico, a eso lo llamamos "tecnología". Cuando el mundo humano tiene como motor 
precisamente la dinámica que se procede del saber y del poder científicotecnológico, a eso lo llamamos revolución científico-tecnológica.

La hipótesis que hoy queremos desarrollar es que lo propio del mundo en el que estamos viviendo se lleva a cabo la más profunda y vertiginosa revolución o cambio radical y científico-tecnológico de la historia de la humanidad desde que esta existe hace ya cerca de 50 mil años. $Y$ que los métodos educativos, las estructuras materiales y administrativas, lo mismo que las políticas públicas en esa materia deben asumir esta realidad como el desafío mayor en la actualidad y darle un sentido humanista con el fin, no solo de preservar la especie y enseñar a las nuevas generaciones a controlarla y al mismo tiempo, profundizarla, sino hacerlo al menor costo posible en lo humano y material.

Porque esta revolución científico-tecnológica provee al ser humano de un poder que nunca antes había tenido. Siendo el poder la idéntica posibilidad de hacer el bien como de hacer el mal, la principal tarea de la educación es en formar en valores, elaborar lúcidamente la escala de los valores, la jerarquía de valores en virtud de la cual puede hacerse del poder que proviene de la revolución científico-tecnológica un factor de crecimiento en lo humano, en otras palabras, se trata de que hacer que el ser humano se humanice cada día mas, sea mas libre, mas racional, mas justo y, en última instancia, mas feliz.

El primer paso para lograrlo es con la toma de conciencia de la realidad que vivimos, lo que caracteriza el mundo que nos entorna y el mundo que corresponderá vivir a las nuevas generaciones, con el fin de que estas puedan lograr avanzar en esta tarea, razón de ser de nuestra existencia sobre la tierra. Comencemos, entonces, por caracterizar esa revolución científico-tecnológica en cuyos seno vivimos sumergidos y de cuyo encauzamiento depende no solo nuestro futuro, sino la sobrevivencia de la especie humana en su conjunto.

Con algunos autores (ver TORRES Martínez, Raúl: Los nuevos paradigmas en la actual revolución científica y tecnológica, EUNED, San José, 2003, p. 4) podemos hablar de tres grandes revoluciones que se han operado en la historia de la civilización universal. La primera operada hace mas o menos unos 10 mil años, fue la que dio origen al neolítico de donde proviene la revolución agrícola. Gracias a ella, la especie logra hacerse sedentaria, se organiza socialmente en aldeas, desarrolla luego la escritura, cultive el pensamiento puro a través de los mitos. Luego vendrá todo lo que solemos llamar historia o memoria escrita de los pueblos.

El surgimiento de la segunda revolución científico tecnológica se inicia con la modernidad occidental a partir del Renacimiento gracias a la 
creación del método científico experimental, su aplicación para explicar los fenómenos d de la naturaleza inanimada en primer lugar, sobre todo en la física y la astronomía (siglos XVI y XVII) y luego su aplicación a las especies vivientes con ciencias como la botánica, la zoología y la clasificación de las especies vivientes (siglos XVIII y XIX) y finalmente su aplicación a los fenómenos propios del mundo de lo humano, como la historia, la lingüística, la sociología, la psicología, la economía y, finalmente, su aplicación a la comunicación humana.

Todo esto se viene produciendo desde el siglo XIX pero se ha acrecentado en extensión y profundidad a medida que avanzó el siglo XX $y$ ahora se ha globalizado y se ha hecho irreversible en forma absoluta y se ha generalizado hasta el punto de que alcanza hasta el mínimo detalle de nuestra vida cotidiana incidiendo incluso en nuestra escala de valores, en nuestra autoconciencia y visión de mundo y en nuestra valoración de la vida en su conjunto. Lo vemos en la vida cotidiana cómo, por ejemplo, Internet constituye la revolución en la comunicación escrita mas grande que se producido en la humanidad después del invento de la imprenta por Gutemberg. También lo vemos en la globalización de la economía, en la mundialización de la política que hace que ya no haya conflictos locales, en la crisis del Estado-nación y el concepto mismo de política que dio origen a la Edad contemporánea después de la Revolución Francesa.

Los ejemplos podrían multiplicarse al infinito, pero eso sería tan solo el aspecto fenomenológico o descriptivo. Debemos aquí profundizar y ver la dimensión epistemológica y su consecuencia en la educación. Por epistemología entendemos en filosofía el estudio de lo que se entiende por saber, razón, ciencia y el uso de los diversos métodos empleados en las distintas ramas de la ciencia, todo desde un enfoque crítico, consistente en verlo todo cuestionándose en qué medida loa diversos métodos cumplen el objetivo de darnos certeza en sus conclusiones, cuáles son los límites y los alcances de los mismos, tanto en las ciencias naturales como humanas y cómo estos criterios de verdad que rigen el método científico han venido evolucionando sobre todo desde que nació la cultura occidental con el surgimiento de la racionalidad helénica.

Según la epistemología actual, existen dos grandes criterios en virtud de los cuales definimos el método científico experimental en general, pero de ahí se derivan métodos específicos para saberes igualmente específicos. La constatación experimental y la formulación de las constantes y variables de los hechos constatados mediante fórmulas algebraicas que lleva a la formulación de leyes científicas. Las leyes científicas se fundan en leyes más universales que llamamos principios. 
Un conjunto de principios lógicamente articulados nos permite elaborar una teoría. Una teoría, por ende, es una explicación universal o generalización cuyas consecuencias lógicas o conclusiones deben ser ratificadas por la observación de hechos debidamente comprobados.

Pero la ciencia no es solo un procedimiento racional, es también e igualmente un fenómeno socio-cultural en la medida en que quienes hacen la ciencia, son seres humanos concretos, hombres y mujeres de una época dada, de una sociedad dada, con creencias, organizaciones políticas y económicas que se rigen por normas no necesariamente científicas sino que responden a necesidades prácticas o costumbres inveteradas. Por eso, cuando un determinado enfoque científico se asume como criterio de verdad en virtud del cual definimos qué es ciencia y qué no es en un determinado momento histórico, a eso se le suele llamar "paradigma" según el filósofo norteamericano Thomas Kuhn. Un cambio en el paradigma es constituye una revolución científico-técnica en su dimensión epistemológica. Por ende, un cambio en los paradigmas científicos es un cambio radical en los criterios en virtud de los cuales definimos qué es ciencia y cuáles sus aplicaciones concretas y consecuencias prácticas en la vida de los seres humanos. Lo que la humanidad entiende por racionalidad cambia con el tiempo y estos cambios producen otros cambios en todos los aspectos de la vida humana. No son solo cambios operados en la mente, sino en la vida de los pueblos, en su manera de organizar la familia, política, la sensibilidad en la creación del arte, de vivir las normas éticas o de concebir sus creencias religiosas.

No nos ha de extrañar, por ende, que también exija enfoques novedosos y métodos distintos en los sistemas educativos que emplea o sigue una determinada sociedad. El pensamiento crítico debe enseñarnos a cuestionarnos sobre las prácticas docentes, la vigencia de las instituciones educativas y los métodos que se siguen para educar sobre todo a las nuevas generaciones en un determinado momento histórico de una determinada sociedad.

Lo que los historiadores de la ciencia y los epistemólogos nos dicen acerca del cambio radical y vertiginoso de los paradigmas científicos que han regido hasta hace un par de generaciones tuvo su origen ya a inicios del siglo pasado. El paradigma que hizo posible la revolución industrial y, con ello, el nacimiento de la edad contemporánea, la democracia formal, el surgimiento del Estado nacional y de la economía capitalista, se llama paradigma mecanicista, basado en la racionalidad cartesiana y en la superioridad epistemológica de la física y la astronomía o modelo galileo- 
newtoniano del universo. Este modelo concibe el mundo como un marco de referencia estable en que la ciencia debe explicar los fenómenos observados como desplazamiento espacial de partículas mediante sistemas de medida o métodos de cuantificación. Se corre el riesgo del reduccionismo, es decir, de explicar causalmente por principios simples lo que en realidad es complejo. Las limitantes epistemológicas de esta concepción se explican porque si la realidad se da dentro del marco del espacio y el tiempo, el paradigma mecanicista o clásico reduce el tiempo al espacio, es decir, niega el tiempo, el devenir, los cambios cualitativos o saltos se reducen a una causalidad lineal.

El nuevo paradigma que hoy rige proviene del concepto de información convertido ahora en categoría, al igual que el de estructura y que provienen tanto de la ciencia biológica como de la lingüística. Pero todo eso solo fue posible porque el paradigma mecanicista entró en crisis con el surgimiento de las leyes de la termodinámica y, en especial, de la segunda ley o de la entropía. La incapacidad para explicar fenómenos como la radiación intermitente del cuerpo negro y la imposibilidad de dar una comprobación experimental a la hipótesis de la existencia del éter concebido como un medio trasmisor de fenómenos como la luz o la energía electromagnética, lo mismo que el descubrimiento de la radioactividad por Henri Becquerel y los esposos Curie en los inicios mismos del siglo $X X$, hicieron posible el surgimiento de las dos grandes teorías que hoy son el marco teórico conceptual en que se desenvuelve la ciencia actual, a saber, la teoría cuántica de Max Planck para explicar los fenómenos de la microfísica y la teoría de la relatividad de Albert Einstein para explicar los fenómenos macrocósmicos de la física.

Esto explica el surgimiento de la teoría de sistemas de Berthalanffi en las ciencias sociales y la cibernética creada por de matemático Norbert Wiener para poner los fundamentos formales de la tecnología actual de la comunicación gracias a la creación de máquinas pensantes, es decir, que forjan relaciones lógico-formales. Con ello van a nacer la s computadoras y hacia 1970 internet. A ello hemos de añadir la explicación química de los procesos biológicos que dan origen a la biología molecular, a un nuevo concepto de ciencia s médicas y, con ello, lo que entendemos por salud, por vida al darse una prolongación de las expectativas de vida que han triplicado o mas en el transcurso del último siglo.

Todo esto podría a primera vista parecer como abstracto y alejado de lo que nos hemos propuesto desarrollar en esta conferencia. En otras palabras, debemos preguntarnos ¿qué tiene esto que ver con la educación y con los sistemas educativos? Para responder a esta pregunta, propongo 
que partamos de lo afirmado en este último párrafo para señalar algunas pautas pero dejando más interrogantes que respuestas, pues lo propio del pensamiento no es acuñar fórmulas, sino abrir horizontes y avizorar nuevas dimensiones, nuevos universos que permitan el crecimiento del ser humano. Algunas conclusiones se imponen.

Una de ellas es revisar nuestro sistema educativo integralmente. Para ello, por lo primero que debemos luchar es por hacer que las políticas educativas sean políticas de Estado y no del gobierno de turno, a fin de evitar que cada cuatro años se improvise dando la impresión de que cada cuatro años comenzamos de cero. Se debe exigir que se hagan realidad las promesas de campaña del candidato ganador a fin de que se cumpla con el presupuesto que la ley exige.

Pero la educación debe ser vista como un espacio de creatividad. El educador no es un funcionario, ni un burócrata, no es un simple empleado sino que hay que volver a la mística de los grandes maestros de inicios de siglo que veían en el educador una vocación, una entrega o dedicación. Se debe cambiar la imagen de los espacios donde se realiza la labor educativa. Las escuelas y colegios no son cárceles, ni las aulas prisiones. Los campos de deporte son tan importantes como las bibliotecas y laboratorios. La función de la educación es despertar la creatividad y todo el entorno debe inducir a ello, tanto en los manuales como en los espacios. Enseñar a leer y a escribir es algo mas que una técnica o una destreza, es ante todo, un salto en el uso de nuestra razón en la medida en que con la lectura y la escritura se debe aprender a aprender a pensar en abstracto, a manejar una interpretación simbólica de lo real que es lo que caracteriza a la razón humana.

A medida que se avanza en el sistema educativo, debe darse una especialización. Así, los liceos de segunda enseñanza beberían destinar los últimos dos años a una mayor especialización, o en ciencias naturales (cosa que se hace con los liceos científicos) liceos humanísticos, artísticos e incluso deportivos. Se debe diversificar la enseñanza en estos últimos años, pero partiendo de una base humanística general que le permita a los educandos adaptarse con facilidad a los cambios que continuamente se operan en todos los saberes científicos: la ciencia no debe ser vista como algo distante sino como parte de nuestra vida cotidiana. Así, se debe aprender a observar nuestro entorno, por ejemplo desarrollar una actitud de conservación ecológica mediante la visita y el estudio de las reservas naturales.

La educación no debe reducirse al aula ni solo a la enseñanza del maestro o profesor. Debe haber charlas, mesas redondas donde participen 
críticamente los educandos. El sistema educativo solo tiene una finalidad: desarrollar la creatividad de los educandos, hacer que tomen conciencia de que solo son seres humanos quienes crean y, al crear, se autocrean. La autoestima, la alegría de saberse creador de algo y creador de sí mismo, es la razón de ser de todo sistema educativo que realmente libere y no enajene.

Pero la enseñanza debe abarcar tanto conocimientos como formación en valores. Por lo que importa tanto el conocimiento de saberes particulares, como empaparse de los valores fundamentales que hagan posible una convivencia civilizada entre todos. Porque construir el ser humano cada día es la tarea fundamental que a todos nos corresponde. El ser humano solo existe si diariamente lo hacemos. No es otra la razón de ser de la educación.

MUCHAS GRACIAS. 